\title{
WORLD FAMILY PORTRAIT
}

\section{The RISC-RISE Consortium}

A World Family Portrait is a joint project of the Consortium for Comparative Research on Regional Integration and Social Cohesion-Social Elevation (RISC-RISE) and Regions $\mathcal{E}$ Cohesion. It aims to promote interdisciplinary and cross-cultural communication through images and essays on the different faces of humanity, including, but not limited to, our similarities and our differences, our strengths and our weaknesses, our hopes and our concerns, our legacies and our aspirations, as well as our interactions with each other and our world. This project seeks to establish a dialogue between human experiences, academic reflections and shared ethics, such as mutual respect, the protection of human dignity and solidarity.

Regions $\mathcal{E}$ Cohesion welcomes submissions of photos and short essays for publication in the World Family Portrait section of the journal. The journal has already published photos and texts on themes such as water, poverty, women, borders, political ideology, democratization, development, religious practices, violence, the environment, and more. Interested contributors should go to https://ojs3.berghahnjournals.com/index .php/reco/login where texts and photographs can be uploaded along with basic information such as contributor name(s), correspondence address, institutional affiliation, and current position. Essays should be approximately 500 words in length. Images should be submitted in a JPEG format at a resolution of approximately $1900 \times 1600$ pixels. All materials must be original. All inquiries and submissions related to RISC-RISE should be sent via the forms on the RISC-RISE Consortium web portal (https://www .uj.ac.za/faculties/humanities/dpir/Pages/risc-rise.aspx). All inquiries and submissions about the World Family Portrait should be sent to reco@ berghahnjournals.com.

\section{In this issue/En este número}

Regions \& Cohesion is proud to present a selection of four photographs submitted by Mauricio Salas Cervantes and taken by Felipe Morales Leal that describe a research visit within a multidisciplinary and multinational project in the transboundary Guatemala-Mexico region with a perspec- 
tive of a landscape analysis. These pictures taken in the Suchiate river in Soconusco region show the complexity and plurality of one of the most transited crossing points between Mexico and Guatemala. We also publish two photographs by our editors Carmen Maganda and Harlan Koff that illustrate their research visit to the Costa Chica Guerrero, Mexico. These photos juxtapose the co-existence of two worlds in this region: one local and one global.

\section{Expresiones paisajísticas de la frontera Guatemala-México en la zona del Soconusco}

Mauricio Pablo Cervantes Salas ${ }^{1}$

A Marcelo, Violeta y Betzabé

La conformación de los Estados Nación está acompañada de una idea abstracta y homogénea de la frontera, y en su función de límite genera dominios para separar y distinguir a unos de otros. No obstante, un análisis paisajístico de la división internacional entre México y Guatemala en la región del Soconusco, ofrece un panorama más complejo, invitando a reflexionar sobre el carácter heterogéneo y plural de las fronteras.

En este trabajo el análisis del paisaje es entendido como la lectura de los elementos físicos, ecológicos y humanos del límite internacional entre México y Guatemala. Este análisis permitió observar que la pluralidad de la frontera descansa en la naturaleza de la apropiación, la diversidad de actores que intervienen en ello, el espacio social y geográfico en el que la apropiación sucede, y la dimensión temporal asociada.

En algunas zonas el control del cruce está en manos de las comunidades locales, y no existe la presencia organizada de personas cobrando o vigilando; la zona es plana y el río es amplio y de escasa profundidad, características que en época de estiaje, permiten cruzar a pie de manera relativamente segura. 


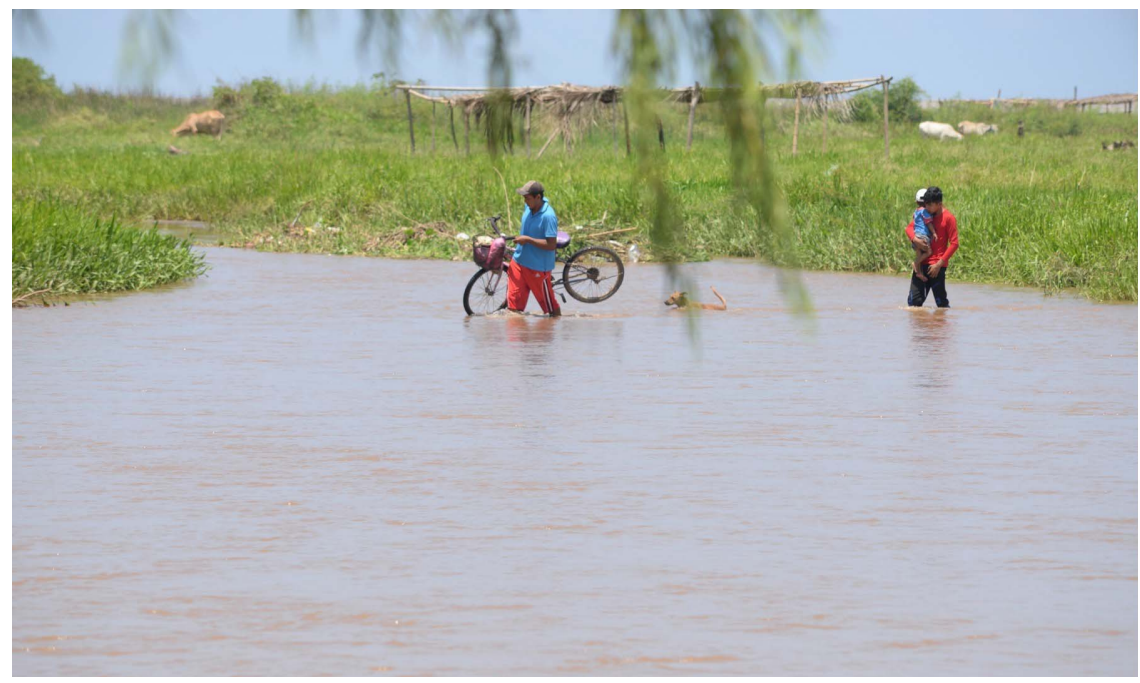

Fotografía de Felipe Morales Leal / 19 mayo 2018 / Río Suchiate / Archivo: Laboratorio Audiovisual de Investigación Social-Instituto Mora

En otros lugares, una hamaca y una mesa sirven para que un cobrador, un supervisor y tres conductores de botes regulen el paso de personas. Para los habitantes locales el río es un lugar de esparcimiento; jóvenes, niños, niñas y mujeres dedican alguna parte de su día a aliviar el sofocante calor del verano.

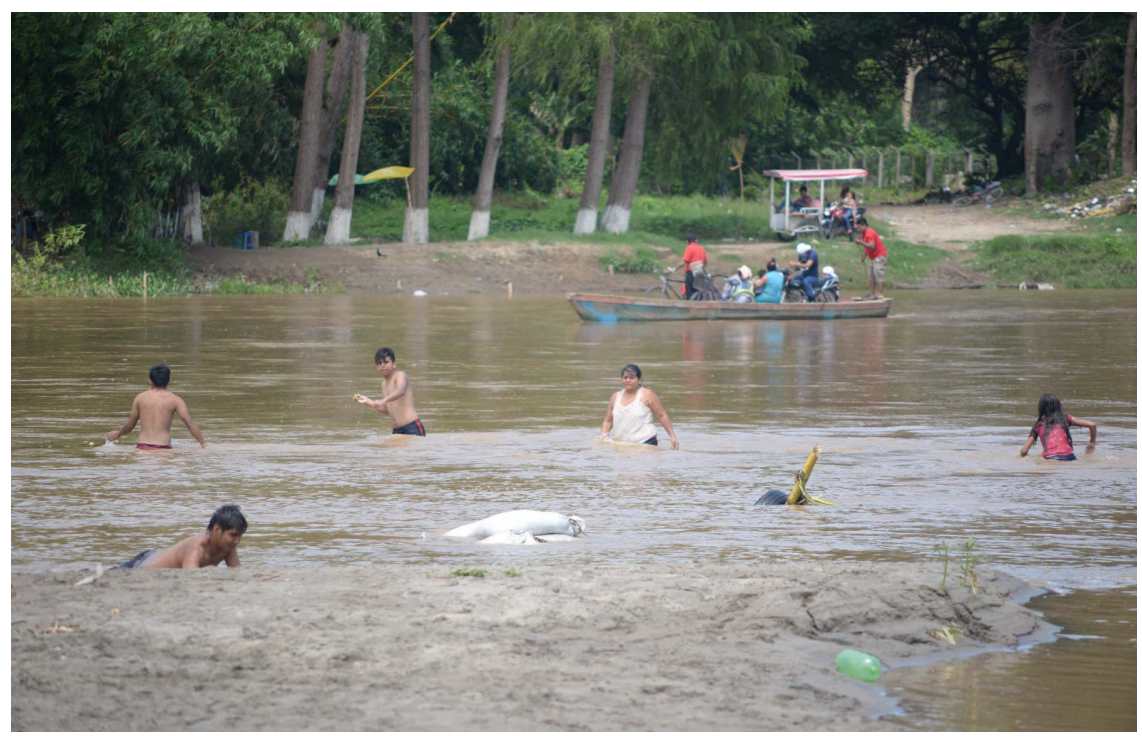

Fotografía de Felipe Morales Leal / 19 mayo 2018 / Río Suchiate / Archivo: Laboratorio Audiovisual de Investigación Social-Instituto Mora 
En Ciudad Hidalgo y Tecún Umán se encuentra una de las zonas de paso más importantes, y en ella conviven el cruce regular e irregular de personas y mercancías. Los dispositivos de control están en manos de organizaciones de "camareros", agrupadas por los dueños, un supervisor, un cobrador y los conductores de las "Cámaras". Después del huracán Stan el río se hizo más ancho y menos profundo, aún así, se ha cobrado la vida de muchas personas que han tratado de atravesarlo a nado.

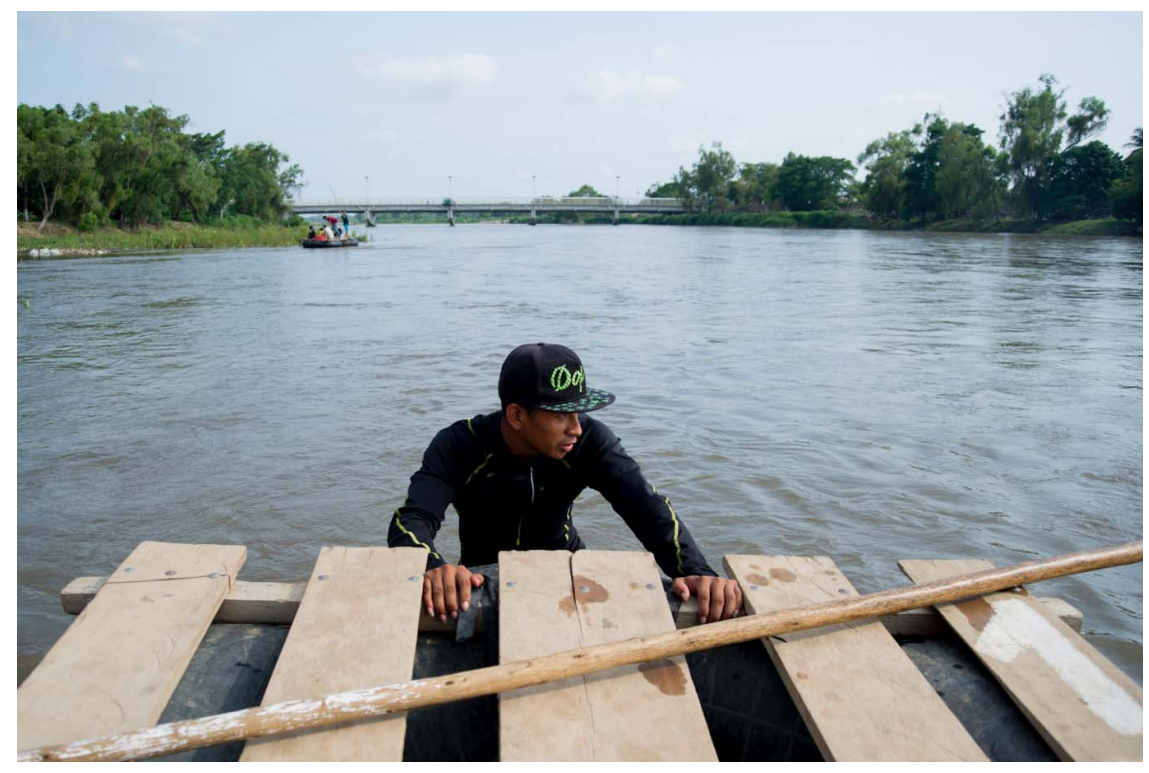

Fotografía de Felipe Morales Leal / 20 mayo 2018 / Río Suchiate / Archivo: Laboratorio Audiovisual de Investigación Social-Instituto Mora

En la aduana El Carmen-El Talismán, el cruce está rodeado de pequeños cerros que le dan forma de embudo, provocando que el flujo se concentre en un espacio reducido. El cruce regular se sucede por el puente internacional y el irregular por debajo de este. A los costados del puente internacional, la disposición de los elementos naturales dificulta el libre tránsito de las personas, obligando a cruzar el río únicamente por este punto. Destaca que en esta zona el Suchiate es más angosto y por ello en época de lluvias su afluente puede concentrar mucha más fuerza. 


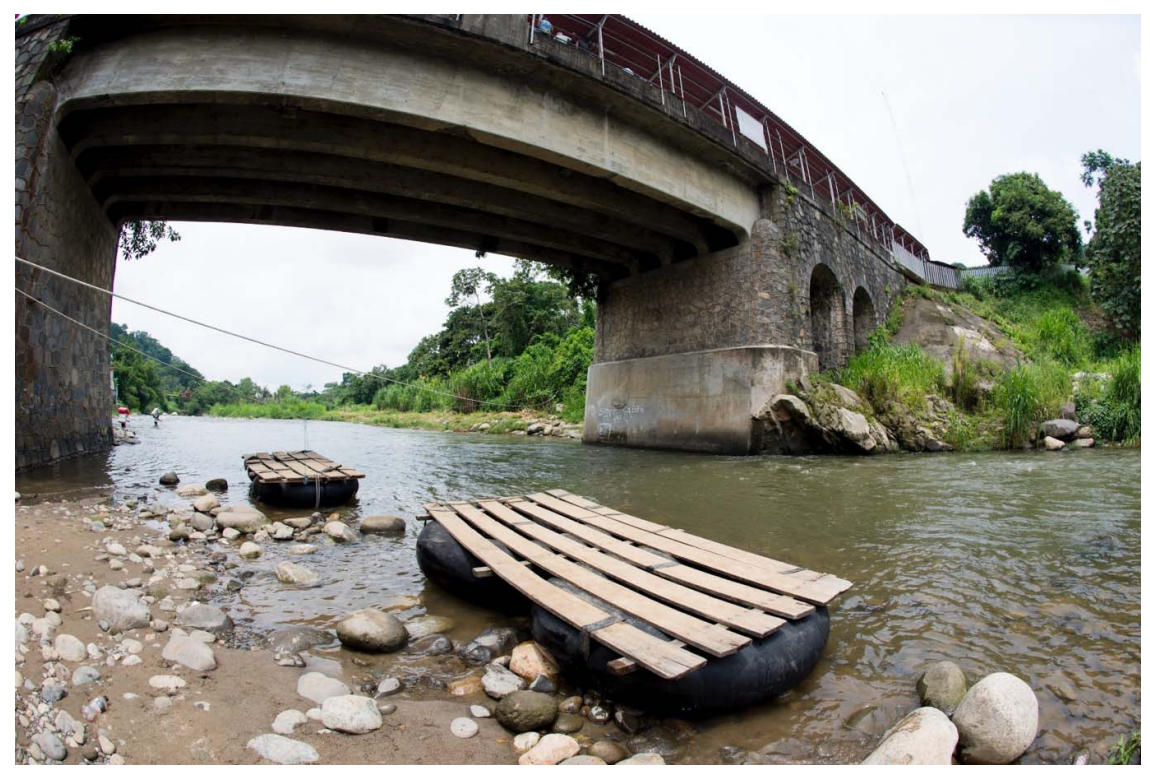

Fotografía de Felipe Morales Leal / 21 mayo 2018 / Río Suchiate / Archivo: Laboratorio Audiovisual de Investigación Social-Instituto Mora

Este trabajo muestra que la conjugación de las lanchas, la expresión geográfica del río, la fuerza de su afluente y la organización social entorno a la vigilancia y control del cruce internacional, son ejemplo de los dispositivos territoriales que facilitan o restringen el flujo de personas a través del río y dotan a la frontera de una alta pluralidad, heterogeneidad y de lo que llamo una "permeabilidad selectiva".

MAURICIO PABLO CERVANTES SALAS es licenciado en biología por la Facultad de Ciencias de la UNAM, maestro en demografía y doctor en estudios de población por El Colegio de México A.C. Actualmente es profesor Investigador del Centro de Investigación en Ciencias de Información Geoespacial A.C. (CentroGeo). En esta institución ha impartido el curso titulado "Espacio y Desigualdad" para la maestría en Planeación Espacial. Sus temas de interés en la investigación están relacionados con desigualdad, familia, territorio y manejo comunitario de recursos naturales. Email: mcervantes@centrogeo.edu.mx 


\section{Un alfabeto, dos universos}

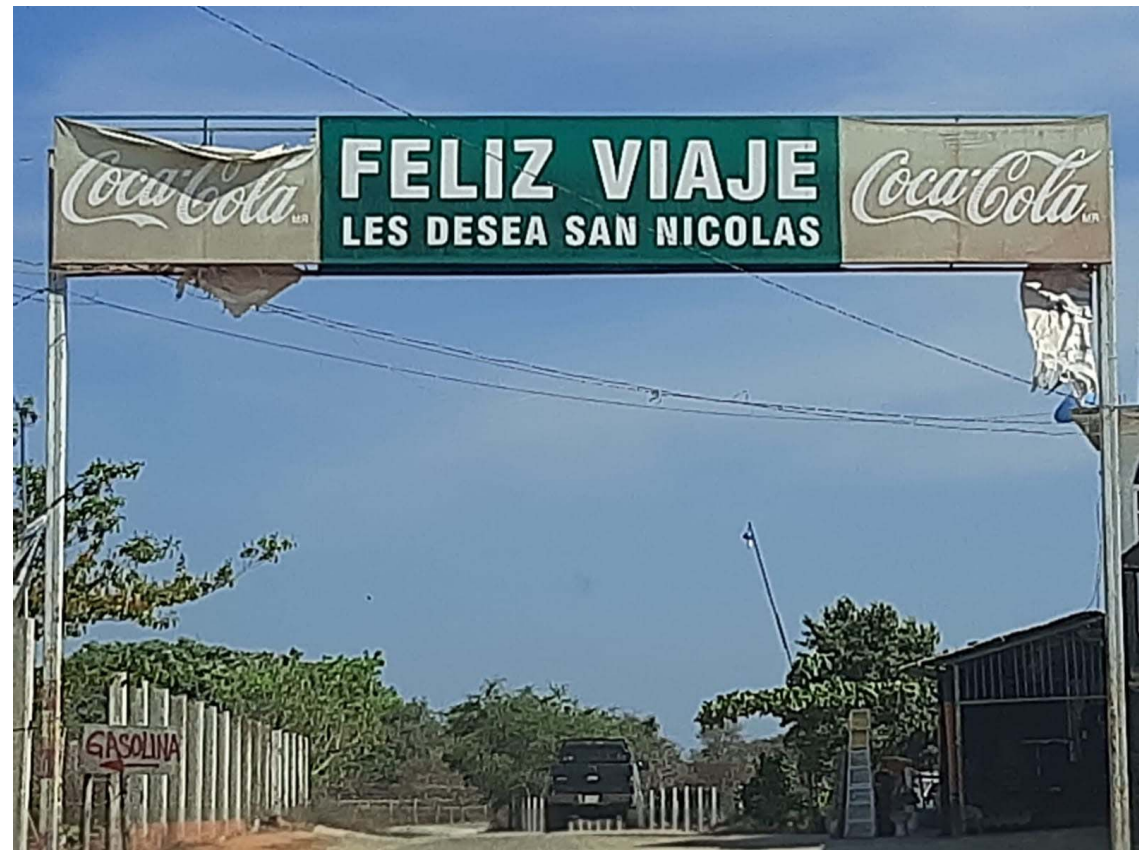

Fotografía de Harlan Koff y Carmen Maganda / marzo de 2021/ San Nicolás, Guerrero

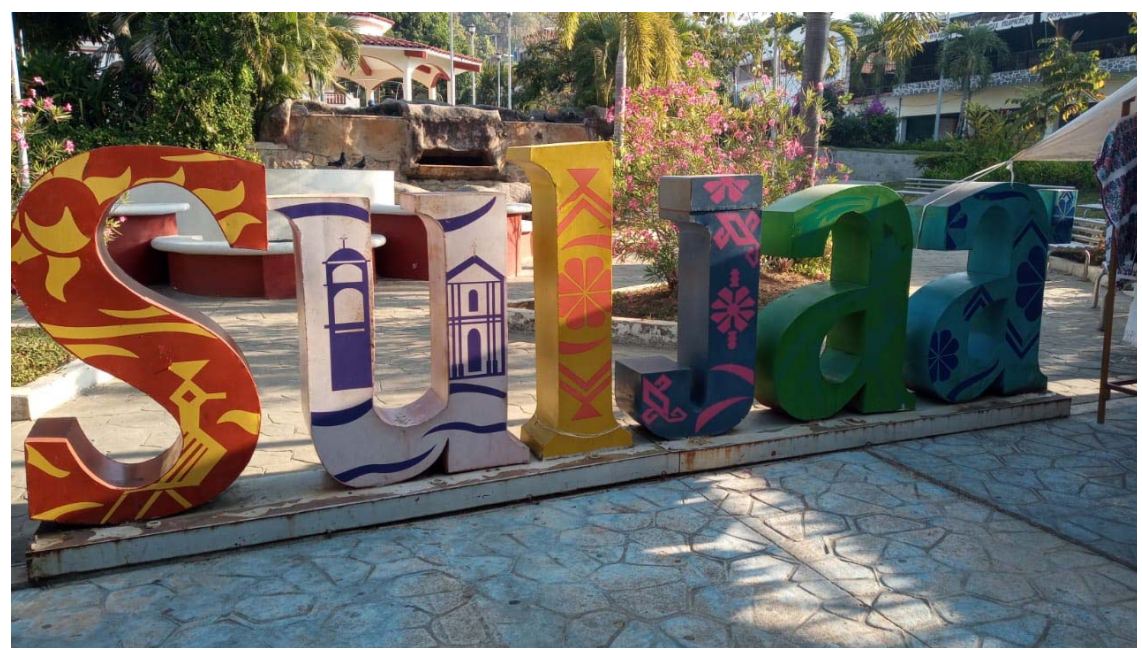

Fotografía de Harlan Koff y Carmen Maganda / marzo de 2021/ Suljaa, Guerrero 
NOTE

1. Este trabajo fue parte del proyectoFORDECYT "Región transfronteriza MéxicoGuatemala: Dimensión regional y bases para su desarrollo integral", apoyado por el Consejo Nacional de Ciencia y Tecnología (Conacyt), solicitud: 291987. 